Original article

\title{
Radiological assessment of surgical treatment results in children and adolescents with pectus excavatum: medium-term results of 75 cases
}

\author{
Sh.K. Khakimov, I.Yu. Khodjanov, Yu.M. Khodjibekova *, Kh.A. Kasymov \\ Traumatologic and Orthopedic Scientific Research Institute of the Republic of Uzbekistan, Tashkent, Uzbekistan \\ * Tashkent Medical Academy, Tashkent, Uzbekistan
}

Received 12 February 2014, Revised 30 May 2014, Accepted 20 June 2014

(C) 2014, Khakimov Sh.K., Khodjanov I.Yu., Khodjibekova Yu.M., Kasymov Kh.A.

C 2014, Russian Open Medical Journal

\begin{abstract}
Objective - Minimally invasive repair of pectus excavatum (PE) has been established as the preferred technique for the repair of PE. Modified techniques of repair based on D. Nuss method for PE are promoted to improve the safety of the procedures. Purpose of this work was to analyzing the operative results with accounting of the toracometric parameters of pectus excavatum in children and adolescents. Methods - Toracometric parameters were the followings, as: sternum rotation, cardiac and pulmonary vein rotation angles, Haller and asymmetry indices in children and adolescents with PE. 48 (64\%) patients are operated by D. Nuss procedure and 27 (36\%) with modifications, due to severity of diagnostic parameters, which were exhibited the significance difference between pre- and postoperative indices, mainly, in patients who has a severe degree changes preoperatively. Results - The sternum rotation degree (in 75 patients) was decreased till $0.4 \pm 0.02^{\circ}(P=0.01)$, whereas was $20.7 \pm 0.46^{\circ}$ preoperatively $(P=0.001)$. Preoperatively, the mean of cardiac rotation angle significantly reduced, preoperative was $56.0 \pm 2.9^{\circ}(P=0.001)$ (in 75 patients) and after operative intervention made up $47.0 \pm 2.7^{\circ}(P=0.001)$. Haller index also is decreased till $2.3 \pm 0.2$, whereas was $3.9 \pm 0.2$ preoperatively $(P=0.002)$. The angle of the pulmonary vein rotation was preoperatively $51.0 \pm 3.4^{\circ}(\mathrm{P}=0.001)$ and after operation was increased till $53.0 \pm 1.8^{\circ}(\mathrm{P}=0.01)$. Conclusions - It has necessity apply the computer tomography investigation in patients with PE deformity. Also, it cannot miss the stage of the sterno-costal complex strength, its pliability and the gravity of the sternum rotation, which may lead to raise the share of good and excellent results.
\end{abstract}

Keywords: pectus excavatum, children, radiological assessment, D. Nuss method

Cite as Khakimov ShK, Khodjanov IYu, Khodjibekova YuM, Kasymov KhA. Radiological assessment of surgical treatment results in children and adolescents with pectus excavatum: medium-term results of 75 cases. Russian Open Medical Journal 2014; 3: 0308.

Correspondence to Sh.K. Khakimov.E.mail: www.sher-fannel-thorax@rambler.ru

\section{Introduction}

The congenital deformations of the chest produce a great problem at the pediatric orthopedics by the severally of its types [1]. Pectus excavatum (PE) is the most common view dysplastic impairment of the chest [2-4], which is characterized with various degrees of the depth of anterior cheat wall, especially in the sternocostal complex area. By domestic authors data the occurrence rate of this pathology was ranged from 0.06 to $2.3 \%$ and by foreign authors literatures was fluctuated from 0.2 to $1.3 \%$, according to the vary of regions [2].

The diagnostic issues of children and adolescents with PE in pre- and postoperative periods were improved their necessity level. Recently, for the better visualization of the chest elements a simple radiography method was considered as a relevant diagnostic method and usually $[5,6], X$-ray radiography was carried out by three planes (antero-posterior projection, lateral view and either left axial or right axial planes).

However, the majority parts of children's anterior chest wall consist of the cartilaginous tissues. Because, the $X$ ray radiography may be low-informative investigative method. The application of Multi Spiral Computed Tomography (MSCT) method in diagnosis of the PE was built the possibility to obtain more information which concern with current pathology [7].

The more representations may obtain in $3 \mathrm{D}$ reconstructions as about the morphological structures of sternocostal complex, the spatial characters of localization and the radiologic condition of the chest inner organs and about the topographic localization of abnormality changed anterior wall parts and mediastinal organs [8].

The current investigation method helps to select of operative treatment approach depending on the angle of sternum rotation and both edges angles of the PE deformity (right and left sides), which they would increase the share of asymmetry. About the standing of the inserted fixation device with appropriate ribs in the correction position of the sternocostal complex in postoperative period was recognized. And also, one of the useful characters of this method was a periodicity of its application with dynamic observation of the deformation development.

Purpose of this investigative work was analyzing the operative results with accounting of the toracometric parameters of pectus excavatum in children and adolescents. 
Table 1. Distribution of patients by sex and method of correction

\begin{tabular}{|c|c|c|c|c|}
\hline \multirow[t]{2}{*}{ Sex } & \multicolumn{4}{|c|}{ Correction method $(n=75)$} \\
\hline & D. Nuss procedure, $n=48(64 \%)$ & The modifications of D. Nuss procedure, $n=27(36 \%)$ & $\mathrm{M} \pm \mathrm{m}$ & P-level (girls vs boys) \\
\hline Girls, $n=25(33.3 \%)$ & $17(22.6 \%)$ & $8(10.7 \%)$ & $12.5 \pm 4.5$ & $<0.05$ \\
\hline Boys, $n=50(66.7 \%)$ & $31(41.4 \%)$ & $19(25.3 \%)$ & $25.0 \pm 6.0$ & $<0.05$ \\
\hline
\end{tabular}

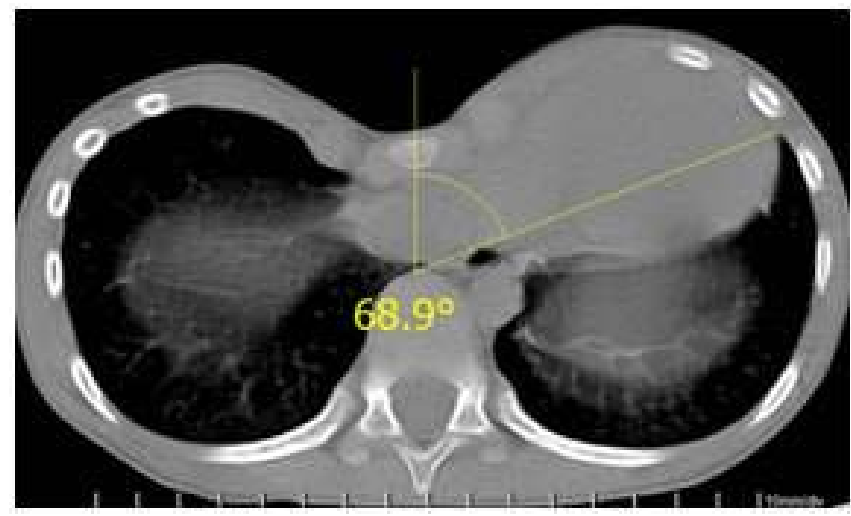

Figure 1. Preoperative axial tomographic scan. The cardiac rotation angle is $68.9^{\circ}$ degrees.

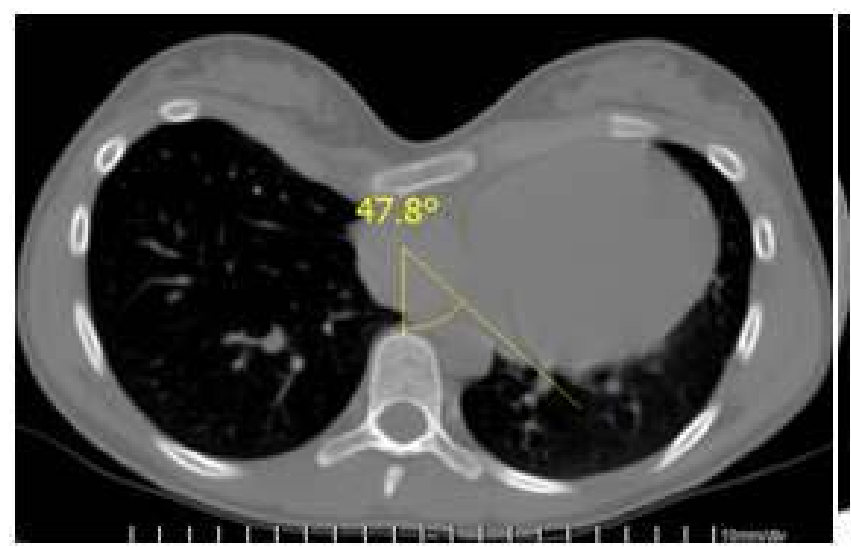

Figure 2. Preoperative axial tomographic scan. The pulmonary vein rotation angle is $47.8^{\circ}$ degrees.

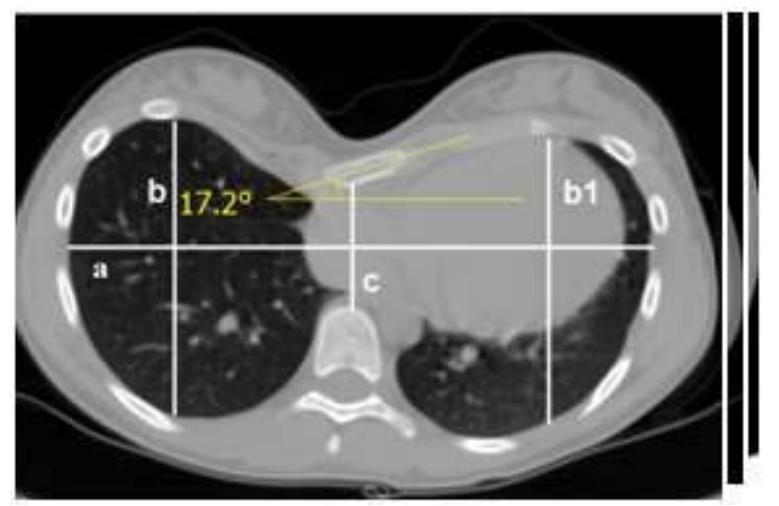

Figure 3. Preoperative axial tomographic scan. The sternum rotation angle is $17.2^{\circ}$ degrees, the parameters of Haller and asymmetry indexes are shown. $a-$ transversal thorax dimension. $b$ - sagittal right and b1 left distances. c - sternovertebral distance.

\section{Material and methods}

The investigation was performed on 75 children, who underwent operative treatment in Children's Traumatology, Deformities of thorax and Spine Pathology department of Scientific Research Institute of Traumatology and Orthopedic of Republic Uzbekistan from 2007 to 2012 years with various severity of pectus excavatum deformity by index Gyzicka. From all patients in 48 (64\%) cases operative intervention was performed by D. Nuss procedure, which was considered as a most Minimal Invasive Repair of Pectus Excavatum (MIRPE) [9-11, 13], that is, the excavated deformity is repaired without any type (wedge or transversal) of the sternotomy and cartilages resection [12] of abnormally developed parts of the ribs and in another 27 (36\%) patients the PE deformation was corrected by modification of D. Nuss procedure and the small statistical validity $(P<0.05)$ was determined between two groups. The patients were distributed by correction method, which is shown in Table 1. Also, patients were distributed through sex, such as boys in $48(64 \%)$ and girls in $27(36 \%)$ cases, with the also small statistical validity between compared groups $(P<0.05)$. Average age was 11 , with ranging from 5 to 17 years. Age-related distribution of patients introduced in the Table 1 too.

\section{MSCT Protocol}

The assessment of efficacy of operative interventions was evaluated with the measuring of the several thoracometric parameters in axial tomographic scan planes of CT investigation, which was carried out in the Roentgen radiology department of Tashkent Medical Academy, with the period from 2007 to 2012 years. Investigation was performed by Siemens Somatom Emothion 62009 (Somaris/5 Syngo CT 2009 E), spiral computer tomography scanner. During the examination, the patient was in supine position and breathing withhold. It is not necessity of preparation for scanning. The hands are behind the head. The scanner diapason ranged from superior thoracic aperture till posterior costodiaphragmatic recess level. The lung window image $\mathrm{W}-/ \mathrm{L}=1500 / 500$, mediastinum without contrast $\mathrm{W}-/ \mathrm{L}=400 / 40.16$ scanners with standard 1-1.5 mm, TF $10 \mathrm{~mm}$, CS $5 \mathrm{~mm}, \mathrm{RI} 4 \mathrm{~mm}$. The scanning parameters were $80-100 \mathrm{kv}$. The slices were performed in sagittal, axial and frontal planes and 3D reconstruction. Tomography slices were led through the most concave chest parts on inhalation and exhalation moments, for determining the difference during the chest movement. The slices were carried out by accuracy in each $1 \mathrm{~mm}$.

There were such indicators measured $[8,14,17]$ during the preand postoperative periods, as: cardiac rotation (Figure 1), pulmonary vein rotation (Figure 2), change of the sternum rotation angle (Figure 3), Haller index (HI) (Figure 3) and asymmetry index (Figure 3).

The degree of cardiac rotation was measured by the angle which made between lines carrying out through midline of the chest to the most lateral and interior point of heart contour. We didn't distribute the obtained cardiac rotation parameters, but may be several normal indices according to the constitution of human body. However, we determined the potential difference these obtained pre- and postoperative parameters. 
Table 2. Results of all parameters of preoperative period

\begin{tabular}{|c|c|c|c|c|c|}
\hline \multirow[b]{2}{*}{ Parameters } & \multicolumn{5}{|c|}{ Severity of deformation } \\
\hline & Mild degree $(n=29)$ & Moderate degree $(n=23)$ & Severe degree $(n=23)$ & All patients $(n=75)$ & P-level \\
\hline Sternum rotation $\left({ }^{\circ}\right)$ & $10.2 \pm 0.5$ & $20.6 \pm 0.7$ & $31.2 \pm 0.2$ & $20.7 \pm 0.5$ & $<0.05$ \\
\hline Haller index & $3.2 \pm 0.2$ & $3.8 \pm 0.2$ & $4.9 \pm 0.3$ & $3.9 \pm 0.2$ & $<0.05$ \\
\hline Cardiac rotation angle $\left({ }^{\circ}\right)$ & $47.0 \pm 3.2$ & $56.0 \pm 2.1$ & $66.0 \pm 3.5$ & $56.0 \pm 2.9$ & $<0.05$ \\
\hline Pulmonary vein angle $\left({ }^{\circ}\right)$ & $60.0 \pm 3.8$ & $52.0 \pm 3.4$ & $43.0 \pm 2.9$ & $51 \pm 3.4$ & $<0.05$ \\
\hline
\end{tabular}

The degree of the inferior pulmonary vein rotation also was measured by the angle which made between lines carrying out through the body of vertebra and across the inferior pulmonary vein. We determined the potential difference these obtained preand postoperative parameters too.

The sternum rotation degree is more important parameter for choosing the correction method. Because, if the osteocartilaginous parts of the chest are yet flexible $[15,16]$, the sternum rotation may be correct itself gradually, during the immobilization period and if the osteocartilaginous parts of the chest are most rigid, the sternum rotation doesn't correct to the end and may occurrence the several secondary atypical deformations. This parameter is measured by the angle between the lines carrying out across the sternum corpus and frontal line of the chest. The chest asymmetry is confirmed according to the degree of sternum rotation and asymmetry index parameters. If the sternum rotation dimension is "low" than $15^{\circ}$, it is "mild", within $15^{\circ}-30^{\circ}$, it is "moderate" degree and "high" than $30^{\circ}$, the deformation is identified as "severe". In figure 3 the sternum rotation angle is averaged as a "moderate" $\left(17.2^{\circ}\right)$.

The asymmetry index (Figure 3 ) is measured on axial chest CT image with dividing by right sagittal diameter to left sagittal (b/b1) and the obtained value is subtracted from 1 . If the obtained value is less than 0.05 the left side of the chest is considered more convex and if the value is bigger than 0.05 the right side is more convex. From all of 75 patients in 41 (54.6\%) was more convex in right side and in other 34 (45.4\%) was left side convexity.

The Haller index (Figure 3 ) has high value to evaluate of the deformation severity. $\mathrm{HI}$ is measured by dividing the transversal chest distance to sternovertebral distance. If $\mathrm{HI}>3.3$ the deformation index is identified as "high" and vice versa, if the dimension is less than 3.3 , the deformation index is considered as a "low".

\section{Methods of correction}

All 75 patients underwent operative treatment. The choice of the correction technique was made due to the intensity of the sternocostal complex elasticity and the gravity of the sternum rotation degree and other indicators, which are used for comparing analysis all of obtained results. The Nuss operation was performed in 48 (64\%) patients, whom the costal part of the chest was maintained its pliability and the mean average of sternal rotation was below $15^{\circ}$. So, we could repair the PE deformations without cartilaginous resection or several types of sternotomy. The excavated deformation is eliminated with the turning over the inserted D. Nuss steel bar. In other 27 (36\%) patients was detected the low degree of the sterno-costal complex elasticity and there was severe degree of the sternum rotation, above $16^{\circ}$. Because, we made the cartilaginous resection on both borders of excavated deformation and transversal wedge sternotomy if the sternum rotation is unidirectional, no so high and the pliable is not absence. If the costal pliable is absence, sternum rotation is multidirectional and too high, the correction was performed with using " $T$ " shaped sternotomy, cartilaginous resection and stabilization the mobilized sterno-costal complex using the D. Nuss steel bar.

\section{Statistical analysis}

Statistical analysis was performed by "STATISTICA" 6.0 software. Pre- and postoperative parameters data were compared according to the t-Student method. Mean values were compared by the method of two independent samples. Postoperative results were assessed by analyzing the same data according to the pair sample method.

\section{Results}

As we cited the diagnostic parameters (cardiac rotation, pulmonary vein rotation, change of the sternum rotation angle, Haller index and asymmetry index) in materials and methods section, their results are shown in Table 2.

Such as it was shown in Table 2 that, the sternum rotation degree of all patients averaged $20.7 \pm 0.5$, with the ranging of deviation from $10.2 \pm 0.5$ till $31.2 \pm 0.7$ in patients of third age groups and fixed P level within 0.05-0.001.

Severity deformation analysis was made with the measuring of Haller index except of Gizycka index and in all patients was averaged $3.9 \pm 0.2$, with the ranging of deviation from $3.2 \pm 0.2$ till $4.9 \pm 0.3, \mathrm{P}$ level was within $0.01-0.002$.

Cardiac rotation and pulmonary vein angles, which are indicating parameters of chest inner organs architectonics dispositions, were changed by the relation to the severity of the PE deformity and during the evaluation it was installed the increase of cardiac rotation degree with the ranging from $47.0 \pm 3.2$ till $66.0 \pm 2.9$ and averaging $56.0 \pm 2.9$ (P level within 0-0.001). Pulmonary vein angle degree has indirect relaion association by the increasing of PE depth and was evaluated the most small meaning in patients with severe degree of $P E-46.0 \pm 2.9$, high meaning was in patients with mild degree of $P E-60.0 \pm 3.8$, averaging $-51.0 \pm 3.4$ and was installed the $P$ level within $0.1-$ 0.001 .

Postoperatively, all (75) patients were examined in the period of 4 years after applying the operative method in $11(14.7 \%)$ patients, $18(24.0 \%)$ patients with 3 years, $26(34,6 \%)$ patients with 2 and other $20(26.7 \%)$ patients with yearly results with introducing above-mentioned parameters. Deformation indices were exhibited the significance difference between pre- and postoperative periods, mainly, in patients who has a severe degree changes preoperatively. Postoperative obtained results are presented in Table 3.

The sternum rotation degree (in 75 patients) was decreased till $0.4 \pm 0.02^{\circ} \quad(P=0.01)$, whereas was $20.7 \pm 0.46^{\circ} \quad(P=0.001)$ preoperatively. In all patients with difference severity of $P E$ deformity was noted significance decreasing of sternum rotation with the ranging from $0.3 \pm 0.03$ till $0.4 \pm 0.02$ ( $P$ level within $0-0.01$ ). 
Table 3. Results of all parameters of postoperative period Parameters

\begin{tabular}{|c|c|c|c|c|c|}
\hline \multirow[t]{2}{*}{ Parameters } & \multicolumn{5}{|c|}{ Severity of deformation } \\
\hline & Mild degree $(n=29)$ & Moderate degree $(n=23)$ & Severe degree $(n=23)$ & All patients $(n=75)$ & P-level \\
\hline Sternum rotation $\left({ }^{\circ}\right)$ & $0.4 \pm 0.02$ & $0.3 \pm 0.03$ & $0.4 \pm 0.02$ & $0.4 \pm 0.02$ & $<0.05$ \\
\hline Haller index & $2.1 \pm 0.2$ & $2.4 \pm 0.3$ & $2.2 \pm 0.1$ & $2.3 \pm 0.2$ & $>0.05$ \\
\hline Cardiac rotation angle $\left({ }^{\circ}\right)$ & $39.0 \pm 2.1$ & $48.0 \pm 2.0$ & $56.0 \pm 2.4$ & $47.0 \pm 2.7$ & $<0.05$ \\
\hline Pulmonary vein angle $\left({ }^{\circ}\right)$ & $61.0 \pm 2.1$ & $53.0 \pm 2.4$ & $46.0 \pm 2.9$ & $53.0 \pm 1.8$ & $<0.05$ \\
\hline
\end{tabular}

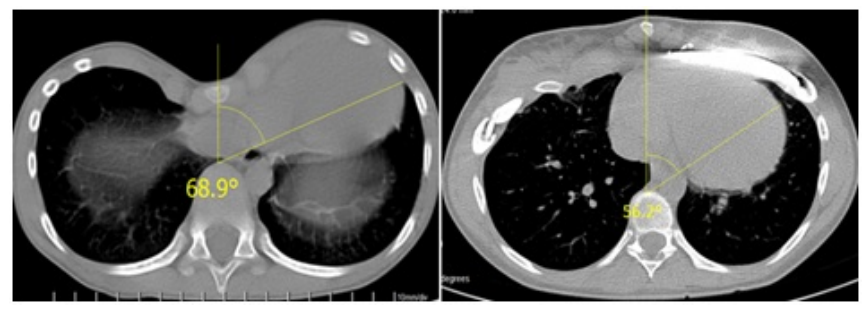

Figure 4. Pre- and postperative axial tomographic images: a) the cardiac rotation angle was $68.9^{\circ}$ preoperatively; b) after correction was decreased till $56.2^{\circ}$. In preoperative image was seen the heart is whole blended to left camera of thorax. After correction the volume of thorax is enlarged and the heart is blended medially and anteriorly. Yearly result.

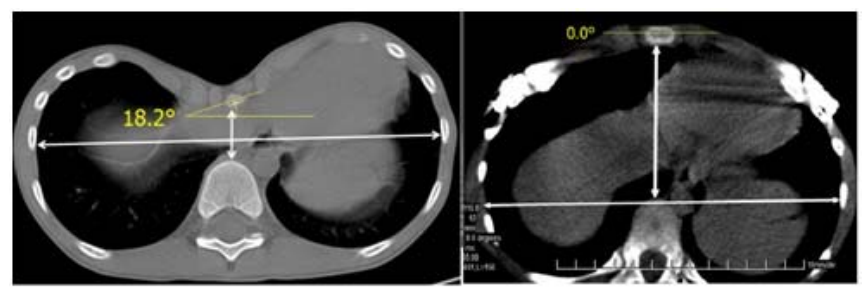

Figure 5. Pre- and postperative axial tomographic images: a) Haller index was 7.79; b) after correction was decreased till 2.46. The sternum rotation was $18.2^{\circ}$ and after correction reduced to $0.0^{\circ} .1 .5$ yearly result.

The mean of cardiac rotation angle significantly reduced, preoperatively $56.0 \pm 2.9^{\circ}(\mathrm{P}-0-0.001$ ) (in 75 patients) with the ranging from $47.0 \pm 3.0$ till $66.0 \pm 3.5$ and after operative intervention made up $47.0 \pm 2.7^{\circ}(P-0.02-0.001)$ fig.4 with the ranging from $39.0 \pm 2.1$ till $56.0 \pm 2.4$. The pulmonary vein angle isn't significantly changed, preoperatively was $51.0 \pm 3.4^{\circ}(P-0.1-0.001)$ (in 75 patients) with the ranging from $60.0 \pm 3.8$ till $43.0 \pm 2.9$ and after operative intervention made up $53.0 \pm 1.8^{\circ}(\mathrm{P}-0-0.01)$ with the ranging from $61.0 \pm 2.1$ till $46.0 \pm 2.9$.

Haller index also is decreased till $2.3 \pm 0.2$ with the ranging from $2.1 \pm 0.2$ till $2.4 \pm 0.3$ without significance, whereas was $3.9 \pm 0.2$ preoperatively $(P-0.01-0.002)$ with the ranging from $3.2 \pm 0.2$ till $4.9 \pm 0.3$ (Figure 5).

\section{Discussion}

Radiological assessment of the chest wall is considered as a fundamental method of evaluation of deformity components. 3D reconstruction enables us to visualization not only chest wall condition but also presents topography condition of inner chest organs. According to the some authors reports the majority of patients give positive results after operative correction in perspective of cosmetic and functional aspects. But, in some situations they become dissatisfied with its cosmetic and functional results. So, we have an opinion, that surgeons must apply the radiological assessment methods occasionally, that the several complaints not should appear and miss.

D. Nuss operation is intended for patients whose deformation is more symmetric, that is, the rotation grade is below than $15^{\circ}$ and the chest is not rigid. If it was performed the Nuss procedure in asymmetric deformation with high range of rotation grade and rigid, it may be appear the secondary atypical deformations in the area or outside from the primary excavated deformation.

Applying the " $T$ " shaped sternotomy is the optimal variant for elimination of the sternum rotation. We could eliminate the PE deformity without any difficulties with using these two types of correction methods.

The proposed indices figured for assessment of obtained postoperative results. As it is known, the deformation is divided by type into two groups: symmetric and asymmetric type of deformations. The choice of the correction method is must be with the accounting of these types of deformations. In several cases the deformation is looked outwardly as a symmetric. But, we may determine true shape of the deformation by the measuring the angle of the sternum rotation, with applying the CT investigation, because the minor sternum rotation is outwardly not visible. So, the correction of these types of the deformations more frequently leads to occurrence the several secondary atypical types of deformations. In primarily cases these negative situations may occur in adolescent aged patients, because their osteocartilaginous parts of the chest are became so rigid. In such cases it is impossible to obtain of satisfactory results with correct chest contour relief. As criteria of the evaluation of like asymmetric type of PE are the sternum torsion and asymmetry index. Preoperatively was more convex in right side in 41 (54.6\%) patients and in other $34(45.4 \%)$ was left side convexity and the average of the sternum rotation angle was $20.7 \pm 0.46^{\circ}(P=0.001)$ in 75 patients preoperatively. We could achieve to decrease till $0.4 \pm 0.02^{\circ}$ postoperatively $(P=0.01)$.

PE deformity is such enlarged, as mechanical pushed away to the chest inner organs. In axial image of CT investigation the stage of the rotation angles of the heart and left inferior pulmonary vein are evaluated. It was the direct correlation between the grades of the sternum rotation and the left inferior pulmonary vein angle with the enlarging of pectus excavatum. So, heart is blended laterally and posteriorly with the rotation in counterclockwise by the development of the deformation, but the angle of the pulmonary vein is decreased. Postoperatively these changes were approximately in norm. Preoperatively the cardiac rotation was $56.0 \pm 2.9^{\circ} \quad(P=0.001)$ and postoperatively averaged $47.0 \pm 2.7^{\circ}$ $(P=0.001)$. The angle of the pulmonary vein rotation was preoperatively $51.0 \pm 3.4^{\circ} \quad(P=0.001)$ and after operation was increased till $53.0 \pm 1.8^{\circ}(\mathrm{P}=0.01)$. It was also enlarged the chest inner volume. The postoperative condition of operated patients is satisfactory and they were not claimed any complaints and were very satisfied with us by cosmetic and functional aspects. 


\section{Conclusion}

It has necessity apply the MSCT investigation in patients with PE deformity in preoperative period by evaluating of the suggested by us indicates. Also, it cannot miss the stage of the sternocostal complex strength. D. Nuss operation is the most optimal correction variant of $\mathrm{PE}$ when the sternocostal complex is sufficiently pliable. It was warranty to obtain the good results if the correction methods will choice according to the aboveadmitted indicates.

Conflict of interest: none declared.

\section{References}

1. Kilda A, Basevicius A, Barauskas V, Lukosevicius S, Ragaisis D. Radiological assessment of children with Pectus excavatum. Indian J Pediatr 2007; 74(2): 143-147. (PMID: 17337826)

2. Vasilenko GP. Pulmonary aspects of conjunctive tissues dysplasia. In: Materials of the VI national congress in devoted to problems pulmonary system (1-4 June 1996). Novosibirsk, 1996. Russian

3. Rudakov SS. The method of combined therapy of funneled deformity of thorax in children with Marfan syndrome and Marfanoid phenotype diseases. Moscow, Russia, 1996; 63 p. Russian

4. Czeizel AE, Hegedus S, Timar L. Congenital abnormalities and indicators of germinal mutations in the vicinity of an acrylonitrile producing factory. Mutat Res 1999; 427(2): 105-123. (PMID: 10393265) (doi: 10.1016/S0027-5107(99)00090-1)

5. Shakirov EA, Satarova FK, et al. Condition of the chest with PE deformity by the data of CT investigation. In: Actual problems of the emergency medical organization: Materials of the $\mathrm{VI}$ Republican conference. Uzb. Urgench, 2006: 591-592.

6. Kanal E. Pregnancy and the safety of magnetic resonance imaging. Magn Reson Imaging Clin N Am 1994; 2(2): 309-317. (PMID: 7489290)

7. Agadjyan VV, Sinica NS, Kornev AN. Our experience in diagnostic and surgical treatment of PE deformity in children and adolescents. Actual problems of traumatology and orthopedics. St-Petersburg, Russia, 2002: 176-177. Russian

8. Chu ZG, Yu JQ, Yang ZG, Peng LQ, Bai HL, Li XM. Correlation between sternal depression and cardiac rotation in pectus excavatum: Evaluation with Helical CT. AJR Am J Roentgenol 2010; 195(1): W76W80. (doi: 10.2214/AJR.09.3199) (PMID: 20566785)

9. Hebra A, Swoveland B, Egbert M, Tagge EP, Georgeson K, Othersen HB $\mathrm{Jr}$, Nuss D. Outcome analysis of minimally invasive repair of pectus excavatum: Review of 251 cases. J Pediatric Surgery 1998; 33: 563-564. (doi: 10.1177/000992280003900913) (PMID: 10693675)

10. Saxena AK, Castellani C, Höllwarth ME. Surgical aspects of thoracoscopy and efficacy of right thoracoscopy in minimally invasive repair of pectus excavatum. J Thorac Cardiovasc Surg 2007; 133(5): 1201-1205. (doi: 10.1016/j.jtcvs.2007.01.040) (PMID: 17467429)

11. Nuss D, Croitoru DP, Kelly RE Jr, Goretsky MJ, Nuss KJ, Gustin TS. Review and discussion of the complications of minimally invasive pectus excavatum repair. Eur J Pediatr Surg 2002; 12: 230-234. (doi: 10.1055/s-2002-34485) (PMID: 12368998)

12. Tang $M$, Nielsen HH, Lesbo M, Frøkiær J, Maagaard M, Pilegaard HK, Hjortdal VE. Improved cardiopulmonary exercise function after modified Nuss operation for pectus excavatum. Eur J Cardiothorac Surg 2012; 41(5): 1063-1067. (doi: 10.1093/ejcts/ezr170) (PMID: 22219453)

13. Nuss D, Kelly RE Jr, Croitoru DP, Katz ME. A 10-year review of a minimally invasive technique for the correction of pectus excavatum. $J$ Pediatr Surg 1998; 33(4): 545-552. (PMID: 9574749) (doi: 10.1016/S0022-3468(98)90314-1)

14. Haje SA, Harcke HT, Bowen JR. Growth disturbance of the sternum and pectus deformities: imaging studies and clinical correlation. Pediatr Radiol 1999; 29(5): 334-341. (PMID: 10382210)
15. Wong $\mathrm{M}$, Carter DR. Mechanical stress and morphogenetic endochondral ossification of the sternum. J Bone Joint Surg Am 1988; 70(7): 992-1000. (PMID: 3403589)

16. Warkany J. Congenital malformations: notes and comments. New York: Yearbook Medical Publ Inc, 1971; 211 p.

17. Haller JA Jr, Kramer SS, Lietman SA. Use of CT scans in selection of patients for pectus excavatum surgery: a preliminary report. $J$ Pediatr Surg 1987; 22(10): 904-906. (PMID: 3681619)

18. Molik KA, Engum SA, Rescorla FJ, West KW, Scherer LR, Grosfeld JL. Pectus excavatum repair: experience with standard and minimall invasive techniques. J Pediatr Surg 2001; 36(2): 324-328. (PMID: 11172426) (doi: 10.1053/jpsu.2001.20707)

\section{Authors:}

Sh.K. Khakimov - PhD, Senior scientific researcher-trainer of the Children's Traumatology, Deformities of Thorax and Spine Pathology Department, Traumatologic and Orthopedic Scientific Research Institute of the Republic of Uzbekistan, Tashkent, Uzbekistan;

I.Yu. Khodjanov - MD, Professor, Chief of the Children's Traumatology, Deformities of Thorax and Spine Pathology Department, Traumatologic and Orthopedic Scientific Research Institute of the Republic of Uzbekistan, Tashkent, Uzbekistan;

Yu.M. Khodjibekova - MD, Associate Professor, Chief of the Roentgen Radiology and Oncology Department, Tashkent Medical Academy, Tashkent, Uzbekistan;

Kh.A. Kasymov - Senior scientific researcher-trainer of the Children's Traumatology, Deformities of Thorax and Spine Pathology Department, Traumatologic and Orthopedic Scientific Research Institute of the Republic of Uzbekistan, Tashkent, Uzbekistan. 